POS PROCEEDINGS

\title{
The ANTARES neutrino telescope
}

\author{
M. Spurio* \\ Dipartimento di Fisica dell'Università di Bologna and INFN-Sezione di Bologna \\ E-mail: maurizio.spurio@unibo.it
}

\section{on behalf of the ANTARES Collaboration}

The ANTARES collaboration has completed since May 2008 the construction of the largest neutrino telescope in the Northern hemisphere. Thanks to its modular geometry ANTARES took data also before completion, since the deployment of its first line. It is installed about $40 \mathrm{~km}$ off the coast of Toulon, France, at a maximum depth of $2475 \mathrm{~m}$ in the Mediterranean Sea $\left(42^{\circ} 48^{\prime} \mathrm{N}, 6^{\circ}\right.$ $10^{\prime} \mathrm{E}$ ). The detector is a 3-dimensional array of photomultiplier tubes (PMTs) distributed along twelve lines. The lines are anchored at the seabed at distances of $\sim 70 \mathrm{~m}$ from each other and tensioned by buoys at the top. Each line has 25 storeys placed every $14.5 \mathrm{~m}$, each of them containing three optical modules (OMs) and a local control module for the corresponding electronics. The 885 OMs containing the PMTs point at $45^{\circ}$ below the horizontal. The total instrumented volume is about $10^{7} \mathrm{~m}^{3}$.

Neutrino telescopes, unlike usual optical telescopes, are "looking downward". The challenge of measuring muon neutrinos via $v_{\mu}+N \rightarrow \mu+X$ consists in reconstructing the muon trajectory using the arrival times and the amplitudes of the Cherenkov light detected by the OMs, and of estimating the muon energy. Up-going muons can only be produced by interactions of up-going neutrinos. Only atmospheric neutrinos that have traversed the Earth represent the irreducible background for the study of cosmic neutrinos. The main goal of the experiment is the detection of high-energy neutrinos from astrophysical sources; other topics include the indirect detection of $v$ 's from dark matter annihilation coming from the Sun direction; the study of the penetrating radiation in the Cosmic Rays. A neutrino telescope in the Northern hemisphere can look at the centre of our Galaxy, and it is complementary to the AMANDA/ICECUBE Antarctic telescope. In parallel, research in several fields of marine sciences will be carried out.

The first results from the ANTARES detector concerning the measurement of the atmospheric muon flux, the searches for point-like neutrino sources and the limits for a diffuse flux of cosmic $v_{\mu}$ are presented.

35th International Conference of High Energy Physics - ICHEP2010,

July 22-28, 2010

Paris France

${ }^{*}$ Speaker. 


\section{Atmospheric muons}

The detection in deep water of cosmic neutrino is not background free. Showers induced by interactions of cosmic rays with the Earth's atmosphere produce atmospheric neutrinos and atmospheric muons. Atmospheric v's that have traversed the Earth represent the irreducible background for the study of cosmic signals. Most of ANTARES events are due to downgoing atmospheric muons, whose flux exceeds that of neutrino-induced events by several orders of magnitude. Atmospheric muons can be a dangerous background for neutrino telescopes, in particular when arriving in bundles, since their Cherenkov light may mimic an upward going muon track in reconstruction programs. On the other hand, atmospheric muons are a useful tool to check the reliability of the ANTARES offline analysis software and for detector calibration. The data collected with the first five lines have been used for a complete study of the major sources of systematic uncertainties in Monte Carlo (MC) simulations [1]. The zenith angle distribution of atmospheric muon events and the vertical muon flux as a function of water depth have been measured. They have been compared with MC expectations obtained with a complete simulation chain and with a parameterized calculation of the underwater muon flux [2] at the detector depth. The results are in agreement, within the systematic uncertainties, with theoretical predictions and previous measurements.

\section{Searches for point like sources}

A search for cosmic $v_{\mu}$ sources has been carried with data collected in 2007 and 2008 corresponding to an integrated live time of 295 days, when the detector grew from 5 to 12 lines. The muon reconstruction algorithm is based on a likelihood fit, which determines the track direction and the goodness of the fit (parameterized by $\Lambda$, a likelihood-based quality variable). $\Lambda$ is used to reject badly atmospheric muons reconstructed as upward-going. For $\Lambda>-5.4$, the median angle between the neutrino direction and the reconstructed one in the selected data sample with a $E^{-2}$ spectrum is $0.5 \pm 0.1$ degree. 2040 events have been found, to be compared with 1093 genuine upgoing neutrinos plus 738 wrongly reconstructed atmospheric $\mu$ expected from MC, Fig. 1 (left).
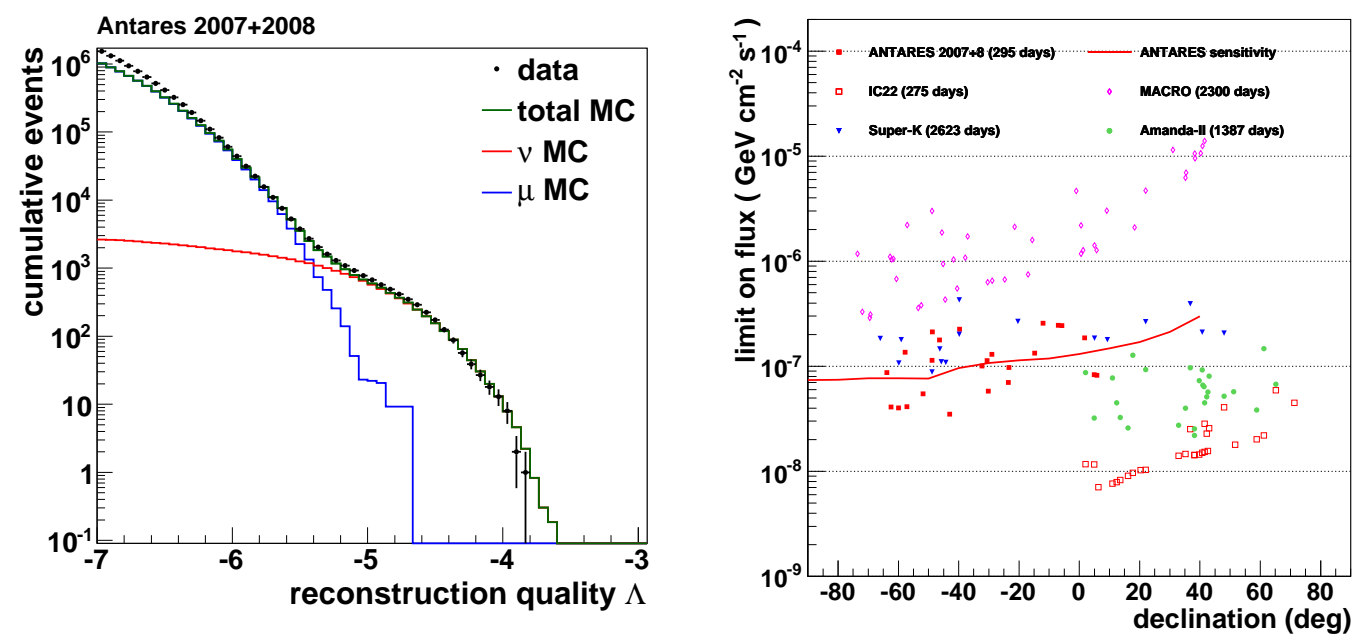

Figure 1: Left: cumulative number of events in data and MC as a function of the $\Lambda$ parameter. Right: $90 \%$ c.l. flux limit for selected sources and ANTARES sensitivity (295 days, 5 to 12 active lines). 
A likelihood ratio method has been used to distinguish between a point source and background fluctuations, and applied to two different searches. The first is a full sky search, that has the sensitivity to discover (at $3 \sigma$ level) sources with a flux greater than $2.2 \times 10^{-7}(\mathrm{E} / \mathrm{GeV})^{-2} \mathrm{GeV}^{-1}$ $\mathrm{cm}^{-2} \mathrm{~s}^{-1}$. The most significant cluster of events is found at r.a. $134.56^{\circ}$ and declination $13.40^{\circ}$. The estimated number of signal events from likelihood fit is 5.2, with a probability of $2.4 \%$ to be compatible with the pure background hypothesis ( $2 \sigma$ one sided). The second is a search around 14 galactic and 10 extra-galactic selected candidates. No significant excess have been found. The limits on the flux from the candidate sources are presented in Fig. 1 (right). The sensitivity for a $E^{-2}$ neutrino spectrum is $7.5 \times 10^{-8} \mathrm{GeV}^{-1} \mathrm{~cm}^{-2} \mathrm{sec}^{-1}$;

\section{Diffuse flux of high-energy $v_{\mu}$}

The prediction of a $v$ flux from extra-galactic sources is a direct consequence of the cosmic ray (CR) and $\gamma$-ray observations. Both electrons and protons can be accelerated by astrophysical objects. If hadrons are accelerated the energy escaping from the sources is distributed between CRs, $\gamma$-rays and neutrinos (see [3] for a review). There are two relevant upper bound predictions [4] for the $v$ diffuse flux: - the Waxman-Bahcall (W\&B), which takes as constraint the CR observations at $E_{C R} \sim 10^{19} \mathrm{eV}$; - the Mannheim-Protheroe-Rachen (MPR) derived using also the observed $\gamma$-ray diffuse flux. For sources transparent to neutrons, the two upper limits are drawn in Fig. 3.

The only way to detect the $v_{\mu}$ diffuse flux is to search for an excess of high-energy events in the measured energy spectrum induced by the atmospheric neutrinos. Cherenkov photons emitted by the muon track and arriving at the OMs without being scattered are referred to as direct photons. For high muon energies $\left(E_{\mu}>1 \mathrm{TeV}\right)$, the contribution of energy losses due to radiative processes increases linearly with $E_{\mu}$ and the resulting electromagnetic showers produce additional light. Scattered Cherenkov radiation or photons originating from electromagnetic showers arriving on the OMs are delayed with respect to the direct photons, with arrival time differences up to hundreds of ns [3]. As a consequence of showers, the percentage of delayed photons with respect to direct photons increases with the muon energy.

To separate atmospheric and astrophysical neutrinos an original energy estimator has been defined; it is based on the average number of hit repetitions (called $R$ ) in the OMs due to the different arrival times of direct and delayed photons. Fig. 2 (left) shows the cumulative distributions of the $R$ variable for expected signal and background [5] neutrinos for 334 days of equivalent live time using at least 9 detection lines (configurations in 2008 and 2009). Using these cumulative distributions, the method [6] has been used on Monte Carlo only to evaluate the value of $R$ which gives the optimal signal to noise ratio, providing the best detector sensitivity. The obtained value is $R=1.31$. The number of expected background atmospheric neutrino events for $R \geq 1.31$ is $10.7 \pm 2$ (including systematic uncertainties). After data un-blinding, 9 events have been found. Fig. 2 (right) shows the distribution of the neutrino candidates as a function of $R$. The corresponding flux upper limit is:

$$
E^{2} \Phi_{90 \%}=5.3 \times 10^{-8} \mathrm{GeV} \mathrm{cm}^{-2} \mathrm{~s}^{-1} \mathrm{sr}^{-1}
$$

This limit holds for a $E^{-2}$ signal spectrum in the energy range between $20 \mathrm{TeV}$ to $2.5 \mathrm{PeV}$, where $90 \%$ of the simulated signal is present. Other models predicting cosmic neutrino fluxes with a spectral shape different from $E^{-2}$ have been tested and some of them excluded at a $90 \%$ c.l. [7]. 

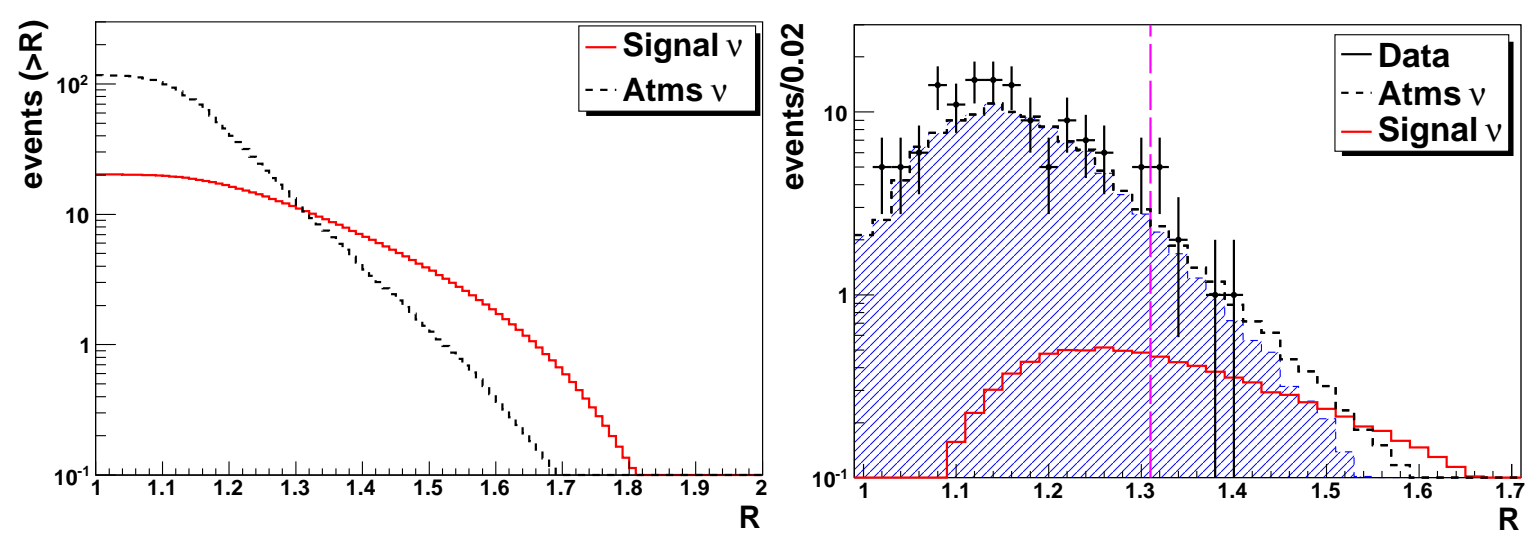

Figure 2: Left: Cumulative distributions of the $R$ variable for simulated diffuse flux signal $\left(E_{v}^{2} \Phi_{v}=\right.$ $\left.1.0 \times 10^{-7} \mathrm{GeV} \mathrm{cm}^{-2} \mathrm{~s}^{-1} \mathrm{sr}^{-1}\right)$ and atmospheric neutrino events [5]. Right: Distribution of the $R$ parameter for the 134 neutrino candidates in the 334 days of equivalent live time. The signal at the level of the upper limit is shown as a full line.

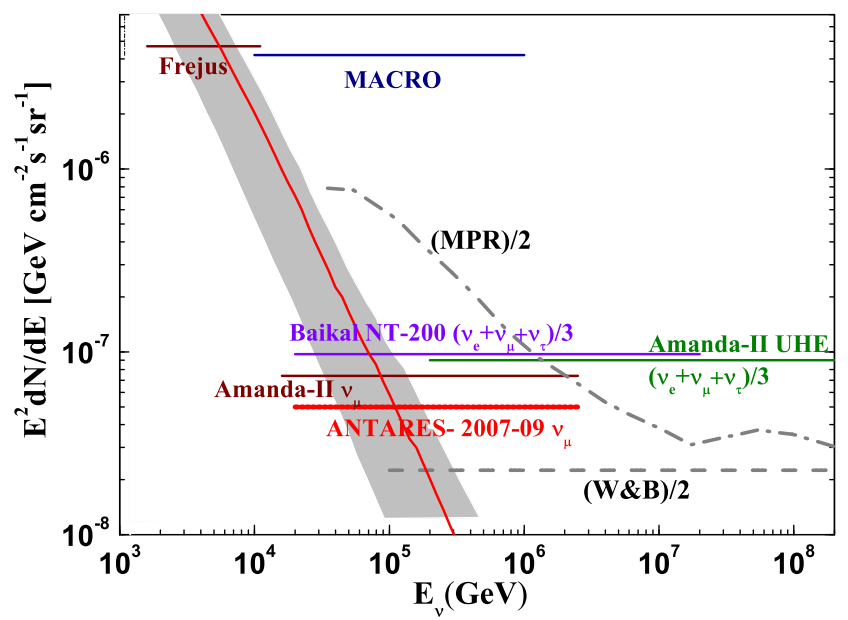

Figure 3: The ANTARES $90 \%$ c.l. upper limit for a $E^{-2}$ diffuse high energy $v_{\mu}+\bar{v}_{\mu}$ flux obtained in this work, compared with the limits from other experiments. The predictions [4] for transparent sources are also shown. The grey band represents the variation from the vertical to the horizontal direction of the atmospheric $v_{\mu}$ flux, the central line the average flux [5].

\section{References}

[1] J.A. Aguilar et al. Astropart. Phys. 34 (2010) 179.

[2] Y. Becherini, A. Margiotta, M. Sioli and M. Spurio, Astropart. Phys. 25 (2006) 1;

G. Carminati, M. Bazzotti, A. Margiotta, and M. Spurio. Comp. Phys. Comm. 179 (2008) 915.

[3] T. Chiarusi and M. Spurio, Eur. Phys. J. C65 (2010) 649.

[4] J. Bahcall, E. Waxman. Phys. Rev. D64(2001) 023002-1;

K. Mannheim, R. J. Protheroe, J. P. Rachen. Phys. Rev. D63 (2000) 023003.

[5] G.D. Barr, T.K. Gaisser, P. Lipari, S. Robbins and T. Stanev. Phys. Rev. D70 (2004) 023006.

[6] G.C. Hill and K. Rawlins, Astropart. Phys. 19 (2003) 393.

[7] J.A. Aguilar et al., Search for a diffuse flux of high-energy $v_{\mu}$ with the ANTARES neutrino telescope. arXiv:1011.3772. Subm. to Phys. Lett. B. 